Pacific Journal of Mathematics

COMPACT FUNCTORS IN CATEGORIES OF
NON-ARCHIMEDEAN BANACH SPACES 


\title{
COMPACT FUNCTORS IN CATEGORIES OF NON-ARCHIMEDEAN BANACH SPACES
}

\author{
KUNG-WEI YANG
}

\begin{abstract}
Let $K$ be a complete, non-archimedean, non-trivially valued field. Let $B$ be the category of all non-archimedean Banach spaces over $K$ satisfying the "condition $(N)$ " with morphisms continuous linear transformations $f,|f| \leqq 1$. In this paper, we first characterize all compact functors $F: B \rightarrow B$ as functors which take finite dimensional spaces to finite dimensional spaces. We then show that in case $K$ is maximally complete the Mityagin-Shvarts imbedding theorem for duals of functors holds true for functors in $B$. Finally, using the above results we show that the dual of a compact functor is itself compact.
\end{abstract}

The present investigation originated in an attempt to apply the Mityagin-Shvarts theory to functors in categories of non-archimedean Banach spaces. In fact, the first and the last results mentioned above are closely related to some problems proposed by Mityagin and Shvarts for functors in categories of ordinary Banach spaces in [4]. Several of these original problems have been solved by a student of mine, Kenneth L. Pothoven, in his thesis [5].

2. Preliminaries. Let $K$ be a complete, non-archimedean, nontrivially valued (i.e., the value group is not trivial) field. We denote by $R$ the valuation ring of $K$ (i.e., the set of all $x \in K$ such that $|x| \leqq 1$ ). A nonarchimedean Banach space over $K$ is a complete normed vector space over $K$ such that the norm satisfies the ultrametric inequality:

$$
|x+y| \leqq \sup (|x|,|y|) \text { for } x, y \in X .
$$

In this paper we shall assume that all the non-archimedean Banach spaces satisfy the following condition [6]. of $K$.

$(N)$ For each $x \in X,|x|$ belongs to the closure of the value group

For the pair $(X, Y)$ of non-archimedean Banach spaces, let $L(X, Y)$ denote the non-archimedean Banach spaces of all continuous linear maps from $X$ to $Y$ with the norm $|f|=\sup \{|f(x)|: x \in X$ and $|x| \leqq 1\}$ (See [6, p. 71]). We let $X^{\prime}$ denote the dual space $L(X, K)$ and for $f: X \rightarrow Y$ in $B$, we let $f^{\prime}: Y^{\prime} \rightarrow X^{\prime}$ denote its dual.

Now we denote by $\boldsymbol{B}$ the category whose objects are non-archimedean Banach spaces over $K$ (satisfying condition $(N)$ ), and whose morphism sets are $B(X, Y)=\{f: f \in L(X, Y),|f| \leqq 1\}$. Clearly, each 
$\boldsymbol{B}(X, Y)$ is an $R$-module.

All (covariant) functors $F: B \rightarrow B$ are assumed to satisfy the following additional conditions:

(1) For each pair $(X, Y)$ of objects in $B$, the induced map $F_{X Y}: B(X, Y) \rightarrow \boldsymbol{B}(F(X), F(Y))$ is $R$-linear, and

(2) For each $f \in \boldsymbol{B}(X, Y),\left|F_{X Y}(f)\right| \leqq|f|$. Such functors are called functors in the category $\boldsymbol{B}$.

A functor $G: B \rightarrow B$ is a subfunctor of $F: B \rightarrow B$ if for each $X$ in $B, G(X)$ is a closed subspace of $F(X)$, and for each $f: X \rightarrow Y$ in $B, G(f): G(X) \rightarrow G(Y)$ is the restriction of $F(f): F(X) \rightarrow F(Y)$ to $G(X)$.

Natural transformations $t: F \rightarrow G$ where $F$ and $G$ are functors in $\boldsymbol{B}$, are assumed to satisfy, in addition to the usual naturality condition, the following conditions:

(1) For each $X$ in $B, t_{X}: F(X) \rightarrow G(X)$ is $K$-linear.

(2) $|t|=\sup \left\{\left|t_{X}\right|: X\right.$ in $\left.B\right\}<\infty$.

Two functors $F$ and $G$ are isometric if there exist natural transformations $t: F \rightarrow G$ and $u: G \rightarrow F$ such that $u \cdot t=1_{F}$ and $t \cdot u=1_{G}$ and for each $X$ in $\boldsymbol{B}, t_{X}$ and $u_{X}$ are isometries. A functor $F$ is isometrically embedded in $G$ if there is a natural transformation establishing an isometry of the functor $F$ and a subfunctor of $G$.

For functors $F$ and $G$ in the category $B$, we denote by $[F, G]$ the class of all natural transformations from $F$ to $G$. Note that if $[F, G]$ is a set, $[F, G]$ has a natural structure of a non-archimedean Banach space with norm defined as in (2) above.

For each $A$ in $B$, we define the functor $\Omega_{A}$ by:

(1) $\Omega_{A}(X)=L(A, X)$, for $X$ in $B$

(2) If $f \in \boldsymbol{B}(X, Y)$, then $\Omega_{A}(f): \Omega_{A}(X) \rightarrow \Omega_{A}(Y)$ is the morphism $\left(\Omega_{A}(f)\right)(g)=f \cdot g$, for $g \in \Omega_{A}(X)$.

For each $A$ in $B$, we define the functor $\Sigma_{A}$ by:

(1) $\Sigma_{A}(X)=A \widehat{\otimes} X($ See $[6, \mathrm{p} .73])$

(2) If $f \in B(X, Y)$, then $\Sigma_{A}(f): \Sigma_{A}(X) \rightarrow \Sigma_{A}(Y)$ is the morphism $1_{A} \widehat{\otimes} f$.

Note that for any $h \in L(A, B)$, there corresponds a natural transformation $\Sigma_{h}: \Sigma_{A} \rightarrow \Sigma_{B}$ defined by:

For each $X$ in $\boldsymbol{B}, \Sigma_{h}: \Sigma_{A} \rightarrow \Sigma_{B}$ is equal to $h \widehat{\otimes} 1_{X} \in L\left(\Sigma_{A} X, \Sigma_{B} X\right)$.

\section{Compact Functors.}

Definition. A functor $F: B \rightarrow B$ is compact (resp. of finite rank), if whenever $f: X \rightarrow Y$ in $\boldsymbol{B}$ is compact (resp. of finite rank), then $F(f): F(X) \rightarrow(F(Y)$ is compact (resp. of finite rank).

Here "compact" means "complètement continus" in $[6, p .72]$, 
and " $f$ is of finite rank" means $\operatorname{dim} f(X)<\infty$.

Lemma 1. For $X$ in $B, 1_{X}: X \rightarrow X$ is compact $\Leftrightarrow \operatorname{dim}(X)<\infty$.

Proof. The assertion follows immediately from [1, Result 2, p.298] by letting $u=1_{X}$ there.

Theorem 1. Let $F: B \rightarrow B$ be a functor. The following are equivalent:

(i) $F$ is compact,

(ii) $F$ takes finite dimensional spaces to finite dimensional spaces,

(iii) $F$ is of finite rank.

Proof. (i) $\Rightarrow$ (ii). Let $F$ be a compact functor. Let $X$ be a finite dimensional space in $B$. By Lemma $1,1_{X}: X \rightarrow X$ is compact. Hence, $1_{F(X)}=F\left(1_{X}\right): F(X) \rightarrow F(X)$ is compact. By Lemma 1 again, we see that $F(X)$ is finite dimensional.

(ii) $\Rightarrow$ (iii). We first note that a morphism $f: X \rightarrow Y$ is of finite rank if and only if $f$ factors through a finite dimensional space $Z$ as in

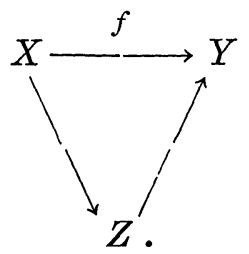

Clearly, if $F$ takes finite dimensional spaces to finite dimensional spaces and if $f$ is of finite rank then $F(f)$ is of finite rank.

(iii) $\Rightarrow$ (i). Let $F: \boldsymbol{B} \rightarrow \boldsymbol{B}$ be a functor of finite rank. Let $f$ : $X \rightarrow Y$ be a compact morphism in $\boldsymbol{B}$. By the definition of compactness of morphisms and by the ultrametric inequality, there exists a sequence of morphisms $f_{n}: X \rightarrow Y$ in $\boldsymbol{B}$ of finite rank converging, in the norm, to $f$. The morphisms $F\left(f_{n}\right): F(X) \rightarrow F(Y)$ are of finite rank and $\left|F\left(f_{n}\right)-F(f)\right| \leqq\left|f_{n}-f\right|$ for $n=1,2, \cdots$. Hence $F(f)$ is compact.

The following corollaries are immediate consequences of Theorem 1.

CoRollary. The following are equivalent:

(i) $A$ in $\boldsymbol{B}$ is finite dimensional,

(ii) The functor $\Sigma_{A}$ is of finite rank,

(iii) The functor $\Sigma_{A}$ is compact,

(iv) The functor $\Omega_{A}$ is of finite rank,

(v) The functor $\Omega_{A}$ is compact.

Definition. A functor $F: B \rightarrow B$ is of null type (type $N$ in [4, 
p. 75]) if $F(K)=0$.

COROLLARY. If a functor $F$ is of null type then it is compact.

4. Duals of compact functors. In addition to all the conditions that are imposed on $K$ in $\S 2$, we shall require throughout this section that the scalar field $K$ is maximally complete. We will continue to use the same letter $\boldsymbol{B}$ to designate the category of all non-archimedean Banach spaces over $K$ satisfying this additional assumption.

Lemma 2. For each $X$ in $\boldsymbol{B}$, the natural morphism $a_{X}: X \rightarrow X^{\prime \prime}$ is an isometric embedding.

Proof. For $x \in X, a_{X}(x)$ is defined by the equation $\left(a_{X}(x)\right)\left(x^{\prime}\right)=x^{\prime}(x)$ for all $x^{\prime} \in X^{\prime}$. Since $X$ satisfies condition $(N)$, for an element $x \in X$ such that $x \neq 0$, we can find a sequence $v_{n}(n=1,2, \cdots)$ of real numbers in the value group of $K$ such that $v_{n} \leqq|x|(n=1,2, \cdots)$ and $v_{n} \rightarrow|x|$. Let $\alpha_{n} \in K$ be chosen so that $\left|\alpha_{n}\right|=v_{n}$. Let $Y$ be the (closed) subspace of $X$ generated by $x$. Define $f_{n}: Y \rightarrow K$ by

$$
f_{n}(\kappa x)=\kappa \alpha_{n}
$$

for $\kappa \in K$. Clearly, $\left|f_{n}\right| \leqq 1$. Since $K$ is maximally complete, we can extend each $f_{n}$ to some $g_{n}: X \rightarrow K$ such that $\left|f_{n}\right|=\left|g_{n}\right|$ ([2]). Now,

$$
\begin{aligned}
& \left|a_{X}(x)\right|=\sup \left\{\left|x^{\prime}(x)\right|:\left|x^{\prime}\right| \leqq 1 \text { and } x^{\prime} \in X^{\prime}\right\} \\
& \quad \geqq \sup \left\{\left|g_{n}(x)\right|: n=1,2, \cdots\right\}=\sup \left\{v_{n}: n=1,2, \cdots\right\}=|x| \cdot
\end{aligned}
$$

On the other hand, clearly we have $\left|a_{X}(x)\right| \leqq|x|$. Hence $a_{X}$ is an isometric embedding.

Definition. The dual functor $D F$ of a functor $F: B \rightarrow B$ is defined by:

(1) For each $A$ in $B, D F(A)=\left[F, \Sigma_{A}\right]$,

(2) For each morphism $e: A \rightarrow B$ in $B, D F(e): D F(A) \rightarrow D F(B)$ is defined by the equation $(D F(e))(t)=\Sigma_{e} \cdot t$.

It will become evident in the course of the proof of Theorem 2 that $D F(A)$ is actually a non-archimedean Banach space.

For a functor $F: B \rightarrow B$, we define the functor $F^{U}: \boldsymbol{B} \rightarrow \boldsymbol{B}$ by:

(1) For each $A$ in $B, F^{U}(A)=\left(F\left(A^{\prime}\right)\right)^{\prime}$

(2) For each morphism $f: A \rightarrow B$ in $B, F^{U}(f)$ is equal to $\left(F\left(f^{\prime}\right)\right)^{\prime}$.

Theorem 2. For any functor $F: B \rightarrow B$, the dual functor $D F$ is isometrically embedded in $F^{U}$. 
Proof. (Mityagain-Shvarts-Linton). Let $\boldsymbol{F}: \boldsymbol{B} \rightarrow \boldsymbol{B}$ be a functor. For each $A$ in $\boldsymbol{B}$, we define the morphism in $\boldsymbol{B}, v_{A}: D F(A) \rightarrow\left(F\left(A^{\prime}\right)\right)^{\prime}$, by: $v_{A}(t)=\operatorname{Tr} \cdot t_{A}^{\prime}$ for $t \in D F(A)=\left[F, \Sigma_{A}\right]$. Here $\operatorname{Tr}$ is the trace map. (Clearly, $\left|v_{A}\right| \leqq 1$ ). We would like to show that, for each $A$, this morphism $v_{A}: D F(A) \rightarrow F^{U}(A)$ is an isometric embedding. To this end, we introduce the functor $\Sigma_{A}^{\prime \prime}$ by setting $\Sigma_{A}^{\prime \prime}(X)=(A \widehat{\otimes} X)^{\prime \prime}$ for $X$ in $\boldsymbol{B}$, and $\Sigma_{A}^{\prime \prime}(f)=\left(1_{A} \widehat{\otimes} f\right)^{\prime \prime}$ for $f: X \rightarrow Y$ in $B$. Let

$$
F^{T}(A)=\left[F, \Sigma_{A}^{\prime \prime}\right] \text {. }
$$

By a proof similar to that of [3, Lemma (4.10), p. 339], we show easily that $F^{T}(A)$ is a set (hence a non-archimedean Banach space). By Lemma $2, A \widehat{\otimes} X$ is isometrically embedded in $(A \widehat{\otimes} X)^{\prime \prime}$. This means that the functor $\Sigma_{A}$ is isometrically embedded in $\Sigma_{A}^{\prime \prime}$. The natural transformation $\Sigma_{A} \rightarrow \Sigma_{A}^{\prime \prime}$ gives rise to an isometric embedding

$$
j_{A}: D F(A) \rightarrow F^{T}(A) \text {. }
$$

The theorem follows immediately from the existence of a morphism $k_{A}$ in $\boldsymbol{B}$ making the following diagram commutative

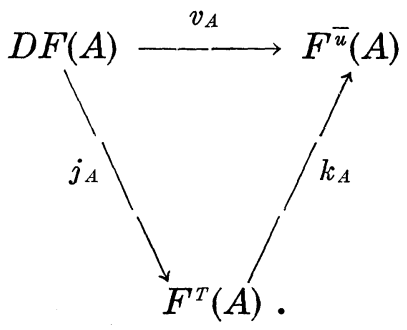

This part of the proof, however, follows exactly the same line of argument as in [3, p. 340-41]. So we shall refrain from repeating the argument here.

CoRollary. If the functor $\boldsymbol{F}: \boldsymbol{B} \rightarrow \boldsymbol{B}$ is of null type, then so is its dual.

THEOREM 3. Let $F: B \rightarrow B$ be a functor. Then:

(i) If $F$ is compact, then so is its dual $D F$.

(ii) If $F$ takes finite dimensional spaces to finite dimensional spaces, then so does its dual DF.

(iii) If $F$ is of finite rank, then so is its dual $D F$.

Proof. In view of Theorem 1, it is sufficient to prove (ii).

Suppose $F$ takes finite dimensional spaces to finite dimensional 
spaces. Let $X$ be a finite dimensional space in $B$. Then $X^{\prime}$ is finite dimensional. By the assumption, $F\left(X^{\prime}\right)$ is finite dimensional. Hence $\left(F\left(X^{\prime}\right)\right)^{\prime}=F^{U}(X)$ is finite dimensional. By Theorem 2, $D F$ is isometrically embedded in $F^{U}$. Obviously, $D F(X)$ is finite dimensional. This completes the proof.

\section{REFERENCES}

1. L. Gruson, Catégories d'espaces de Banach ultramétriques, Bull. Soc. Math. France, 94 (1966), 287-299.

2. A. W. Ingleton, The Hahn-Banach theorem for non-archimedean valued fields, Proc. Cambridge Phil. Soc., 48 (1952), 41-45.

3. F. E. J. Linton, Autonomous categories and duality of functors, J. of Algebra, 2 (1965), 315-349.

4. B. S. Mityagin and A. S. Shvarts, Functors in categories of Banach spaces, Russian Math. Surveys, 19 (1964), 65-127.

5. K. L. Pothoven, Compact functors and their duals in categories of Banach spaces, Trans. Amer. Math. Soc., (to appear).

6. J.-P. Serre, Endomorphismes complètement continus des espaces de Banach p-adiques, Institut des Hautes Études Scientifiques, Publications Mathématiques, 12 (1966), 69-85.

Received August 19, 1970. This work was supported by a Faculty Research Fellowship from Western Michigan University.

WESTERN MICHIGAN UNIVERSITY 


\title{
PACIFIC JOURNAL OF MATHEMATICS
}

\author{
EDITORS
}

\author{
H. SAMELSON \\ Stanford University \\ Stanford, California 94305 \\ C. R. HOBBY \\ University of Washington \\ Seattle, Washington 98105
}

J. DugundJI

Department of Mathematics

University of Southern California

Los Angeles, California 90007

RICHARD ARENS

University of California

Los Angeles, California 90024

\section{ASSOCIATE EDITORS}
E. F. BeCKenBaCH
B. H. NeUMANN
F. WOLF
K. Yoshida

\section{SUPPORTING INSTITUTIONS}

\author{
UNIVERSITY OF BRITISH COLUMBIA \\ CALIFORNIA INSTITUTE OF TECHNOLOGY \\ UNIVERSITY OF CALIFORNIA \\ MONTANA STATE UNIVERSITY \\ UNIVERSITY OF NEVADA \\ NEW MEXICO STATE UNIVERSITY \\ OREGON STATE UNIVERSITY \\ UNIVERSITY OF OREGON \\ OSAKA UNIVERSITY
}

\author{
UNIVERSITY OF SOUTHERN CALIFORNIA \\ STANFORD UNIVERSITY \\ UNIVERSITY OF TOKYO \\ UNIVERSITY OF UTAH \\ WASHINGTON STATE UNIVERSITY \\ UNIVERSITY OF WASHINGTON \\ AMERICAN MATHEMATICAL SOCIETY \\ NAVAL WEAPONS CENTER
}

The Supporting Institutions listed above contribute to the cost of publication of this Journal, but they are not owners or publishers and have no responsibility for its content or policies.

Mathematical papers intended for publication in the Pacific Journal of Mathematics should be in typed form or offset-reproduced, (not dittoed), double spaced with large margins. Underline Greek letters in red, German in green, and script in blue. The first paragraph or two must be capable of being used separately as a synopsis of the entire paper. The editorial "we" must not be used in the synopsis, and items of the bibliography should not be cited there unless absolutely necessary, in which case they must be identified by author and Journal, rather than by item number. Manuscripts, in duplicate if possible, may be sent to any one of the four editors. Please classify according to the scheme of Math. Rev. Index to Vol. 39. All other communications to the editors should be addressed to the managing editor, Richard Arens, University of California, Los Angeles, California, 90024.

50 reprints are provided free for each article; additional copies may be obtained at cost in multiples of 50 .

The Pacific Journal of Mathematics is published monthly. Effective with Volume 16 the price per volume (3 numbers) is $\$ 8.00$; single issues, $\$ 3.00$. Special price for current issues to individual faculty members of supporting institutions and to individual members of the American Mathematical Society: $\$ 4.00$ per volume; single issues $\$ 1.50$. Back numbers are available.

Subscriptions, orders for back numbers, and changes of address should be sent to Pacific Journal of Mathematics, 103 Highland Boulevard, Berkeley, California, 94708.

PUBLISHED BY PACIFIC JOURNAL OF MATHEMATICS, A NON-PROFIT CORPORATION

Printed at Kokusai Bunken Insatsusha (International Academic Printing Co., Ltd.), 270, 3chome Totsuka-cho, Shinjuku-ku, Tokyo 160, Japan. 


\section{Pacific Journal of Mathematics}

\section{Vol. 39, No. $3 \quad$ July, 1971}

William O'Bannon Alltop, 5-designs in affine spaces ................... 547

B. G. Basmaji, Real-valued characters of metacyclic groups ................. 553

Miroslav Benda, On saturated reduced products....................... 557

J. T. Borrego, Haskell Cohen and Esmond Ernest Devun, Uniquely representable semigroups. II.......................................

George Lee Cain Jr. and Mohammed Zuhair Zaki Nashed, Fixed points and stability for a sum of two operators in locally convex spaces ....................

Donald Richard Chalice, Restrictions of Banach function spaces ...............

Eugene Frank Cornelius, Jr., A generalization of separable groups ..............

Joel L. Cunningham, Primes in products of rings ......................

Robert Alan Morris, On the Brauer group of $Z$.

593

603

615

David Earl Dobbs, Amitsur cohomology of algebraic number rings ...............

Charles F. Dunkl and Donald Edward Ramirez, Fourier-Stieltjes transforms and

weakly almost periodic functionals for compact groups ...................

Hicham Fakhoury, Structures uniformes faibles sur une classe de cônes et

d'ensembles convexes ......................................

Leslie R. Fletcher, A note on C $\theta \theta$-groups.

Humphrey Sek-Ching Fong and Louis Sucheston, On the ratio ergodic theorem for

semi-groups............................................

James Arthur Gerhard, Subdirectly irreducible idempotent semigroups ...........

Thomas Eric Hall, Orthodox semigroups.....................

Marcel Herzog, $C \theta \theta$-groups involving no Suzuki groups ..........

669

687

John Walter Hinrichsen, Concerning web-like continua ..........

691

Frank Norris Huggins, A generalization of a theorem of F. Riesz.

695

Carlos Johnson, Jr., On certain poset and semilattice homomorphisms

703

Alan Leslie Lambert, Strictly cyclic operator algebras ...........

717

Howard Wilson Lambert, Planar surfaces in knot manifolds . . .

727

Robert Allen McCoy, Groups of homeomorphisms of normed linear spaces ....... 735

T. S. Nanjundiah, Refinements of Wallis's estimate and their generalizations ...... 745

Roger David Nussbaum, A geometric approach to the fixed point index .......... 751

John Emanuel de Pillis, Convexity properties of a generalized numerical range .... 767

Donald C. Ramsey, Generating monomials for finite semigroups ....

783

William T. Reid, A disconjugacy criterion for higher order linear vector differential equations...

Roger Allen Wiegand, Modules over universal regular rings...

Kung-Wei Yang, Compact functors in categories of non-archimedean Banach

spaces.

R. Grant Woods, Correction to: "Co-absolutes of remainders of Stone-Čech compactifications".

Ronald Owen Fulp, Correction to: "Tensor and torsion products of

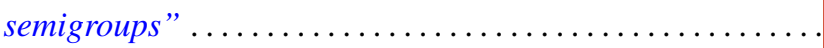

Bruce Alan Barnes, Correction to: "Banach algebras which are ideals in a banach 